\title{
Mathematical Representation of Color Spaces and Its Role in Communication Systems
}

\author{
Riyadh M. Al-saleem $\mathbb{D}^{1},{ }^{1}$ Baraa M. Al-Hilali $\mathbb{D}^{2},{ }^{2}$ and Izz K. Abboud $\mathbb{D}^{3}$ \\ ${ }^{1}$ College of Engineering, University of Samarra, Iraq \\ ${ }^{2}$ College of Education, University of Samara, Iraq \\ ${ }^{3}$ Computer Engineering Department, Mustansiriyah University, Iraq
}

Correspondence should be addressed to Riyadh M. Al-saleem; alsaleem1962@yahoo.com

Received 21 January 2020; Accepted 23 March 2020; Published 18 May 2020

Academic Editor: Sheng Zhang

Copyright (c) 2020 Riyadh M. Al-saleem et al. This is an open access article distributed under the Creative Commons Attribution License, which permits unrestricted use, distribution, and reproduction in any medium, provided the original work is properly cited.

\begin{abstract}
In this research, the color system was analyzed according to the International Lighting Commission (CIE) standard by studying the theoretical aspect of the general color system according to the latest communication theories of the visual image supported by mathematical equations and illustrations supporting the relevant hypotheses and then a comparison of evolution. There is an enormous use in color spaces and systems according to their own use, where the use of colors has become in various areas including television systems; computer systems; industry and product colors; printing of all kinds of black and white and color; paint colors for buildings, houses, and cities; and many other color-related uses. The development of color spaces and systems from 1931 to the present day has expanded a lot, and this led to the emergence of new areas of color systems characterized by accuracy and beauty and became the color of the subcolors according to the desire of the customer and the quality of use. The development of color systems has an impact on visual communication such as television broadcasting systems, medical image processing, and video signal processing, as well as in the field of computer such as graphic equipment and printing.
\end{abstract}

\section{Introduction}

Color is perceived as a phenomenon and not a natural dimension such as length or temperature, but the electromagnetic ray of the visible wavelength is measurable as a natural quantity, so there is an appropriate form of representation for mathematical requirements. In human color, these different requirements cannot meet both, which is why the representation used varies according to the target of processing in the application used. Color spaces refer to harmonized systems in which values are represented by the standard color scheme in accordance with the International Lighting Commission (CIE) standard. The color spaces can be extracted from "red color, green color, and blue color" (RGB) information, the three primary colors that cannot be dealt with in mathematical and technical processors. It does not give an accurate description of the rest of the resulting colors, so fragmentation of the image was used to the original state without colors, i.e., black and white (luminance $(\mathrm{Y})$ ), and is treated as a light intensity based on the grayscale between black and white as in the case of television broadcasting is modulated (modulation) separately. The colors are in two vehicles carrying their characteristics accurately (chrominance) compatible with the color scheme and can be processed mathematically and then the formation of an electronic circuit to achieve this, and in the case of television broadcasting, it is assigned a secondary carrier (sub) working package area.

An international forum was established under the name of the International Lighting Committee (CIE), which is in fact a development of the Maxwell triangle to determine the distribution and mixing of colors for the purpose of dealing with them to switch between operating systems of various measurements, including television systems (NTSC, PAL, and SECAM). It is compatible with the system, for example, the National Television System Committee (NTSC) which is used in the USA and Japan; the Phase Alternating Line (PAL) system in most of Europe; the sequential color with memory (SECAM) system in France; printing systems cyan color, 
magenta color, yellow color, and black color (CMYK); HSV (hue, saturation, value); and others, as well as RGB main color branches.

The process of color processing, accuracy, and purity depends on the nature of the basic components of the image and methods of representation mathematically and technically in the transmitter and receiver, making it identical to the original natural state inspired by them, and that the default state is the lighting element and two color characteristics, and to add other complementary compounds which can lead to a significant improvement in performance is offset by an increase in the complexity of mathematical representation and then technical output (as in the case of adaptive color space) [1].

\section{Historical Background}

Founded in 1913 and headquartered in Vienna, the capital of Austria, the International Commission is mandated by the standards of light, lighting, color, and color space. The organization consists of seven sections; each technical committee implements its program under the supervision of the director of the department; these sections are vision and color, light and measuring radiation, lighting design and internal environment, signal lighting, transportation, outdoor lighting, and other applications, biology, chemistry, optical light, and imaging technology.

Six years after the founding of the Optical Society of America, the International Lighting Association met at its eighth session in 1931 to develop an international characterization and compatibility of colorimetric measurements and to update the recommendations made by the American Optical Society based on development efforts within ten years. The meeting at the University of Cambridge developed a definition of XYZ color space, and standard illuminate types A, $\mathrm{B}$, and $\mathrm{C}[2]$.

\section{Methods}

In this research, the evolution of the mathematical representation of color spaces has been analyzed. This development has led to applications in the field of the use of colors in images, videos, the development of television broadcasting systems, and the emergence of digital television and films as well as the uses of the Internet, i.e., the supreme value of this development is the evolution in the field of analogue and digital communications; then, a model was chosen for one of the spaces and analyzed by the Matlab program to show the accuracy of the mathematical representation the segmentation of the color system into its main properties, which is the gray gradient, as well as the color characteristics resulting from its mixing operations with mathematical proportions for each type.

\section{Color Space XYZ 1931 CIE}

XYZ 1931 CIE (also known as 1931 CIE) is one of the first mathematical definitions of chromatic sensation, developed by the International Lighting Authority in 1931.

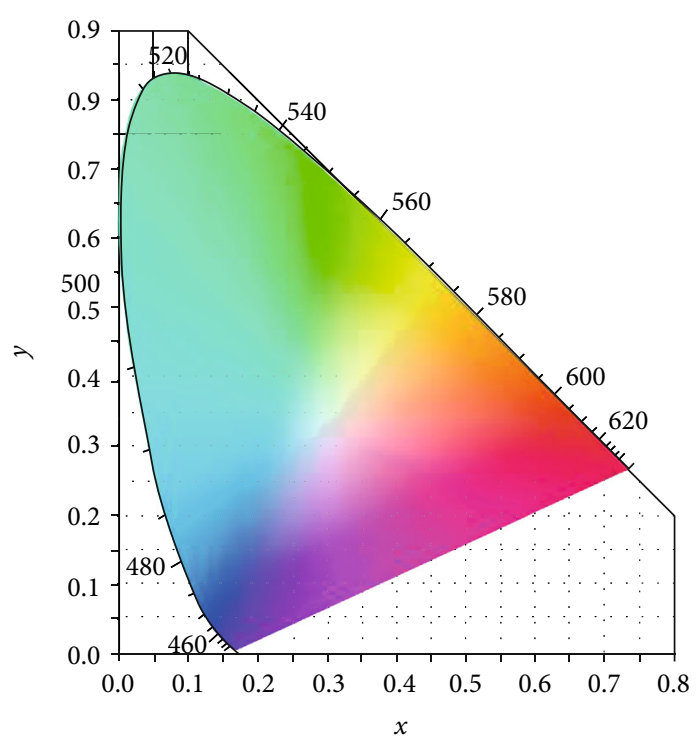

Figure 1: 1931 CIE [2].

The "CIE" color space (Figure 1) is derived from a series of experiments by George Wright in 1920, and the combined results of experimental specifications including RGB CIE triincentive spectral colors (XYZ) color space were derived.

We can define the color of the wavelength $(\lambda)$ as follows:

$$
\begin{aligned}
& X=C \lambda, \\
& x=\frac{X}{X, Y, Z},
\end{aligned}
$$

where $x$ is the color we want to get it and $C$ is the constant multiplied by length which is the speed of light.

We now need only two coordinates $(X, Y)$ to calculate the third set. The values $X, Y$ are defined as the color characteristic [2].

Distinguish colors by luminance parameter $(X)$ and coordinate chromatin $(X, Y)$. $(X, Y$, and $Z)$ is the triple emitting, which gives incentive values (i.e., force) for all three basic lights in red and green needed to match the color blue $[2,3]$ :

$$
\begin{aligned}
& x=\frac{X}{X+Y+Z}, \\
& y=\frac{Y}{X+Y+Z}, \\
& z=\frac{Z}{X+Y+Z=1-x-y} .
\end{aligned}
$$

The red and green colors found large values of $(x, y)$ sequentially, and small values of $(X, Y)$ or near the centre point of the color chart. Therefore, the mixing grades are changed depending on the location of the rendezvous point according to their location to the main colors, because the presence of advanced modern techniques in computer science has led to the emergence of too many gradients of the same color [4]. 
4.1. CIEUVW. "CIEUVW" color space, also known as the CIE $1964\left(U^{*}, V^{*}, W^{*}\right)$ :

$$
\begin{aligned}
U^{*} & =13 W^{*}\left(u-u_{0}\right), \\
V^{*} & =13 W^{*}\left(v-v_{0}\right), \\
W^{*} & =25 Y^{1 / 3}-17,
\end{aligned}
$$

where $\left(U_{0}, V_{0}\right)$ is white and $Y$ point is the value of the luminous object.

" $U V W$ " is the color space in order to be able to calculate the color differences without the need for a constant setting luminance, $W^{*}$ is the definition of lightness indicator, and the components of color $U^{*}$ and $V^{*}$ are defined so that the white point maps to the original; this arrangement has the advantage of being able to express positions of tones with constant saturation simply as $\left\{\left(U^{*}\right) 2+(\right.$ $\left.V^{*}\right) 2=C$ \} fixed $C[5]$.

4.2. CIELUV. "CIELUV" is the color space by the International Committee that adopted lighting (CIE) in 1976 $\left(L^{*}, u^{*}, v^{*}\right.$ color space) that is used extensively for applications such as computer graphics, which deal with colored lights. Although mixtures added of different colored lights will fall on the line in the diagram color uniform CIELUV in the so-called CIE 1976 UCS, these mixtures added will not be contrary to popular belief, located along the line in the space CIELUV color unless mixture is fixed in the lightness [6].

$$
\begin{aligned}
& L^{*}=\left(\frac{29}{3}\right)^{3} \frac{Y}{Y n}, \quad \frac{Y}{Y n} \leq\left(\frac{6}{29}\right)^{3}, \\
& L^{*}=116\left(\frac{Y}{Y n}\right)^{1 / 3}-16, \quad \frac{Y}{Y n}>\left(\frac{6}{29}\right)^{3}, \\
& u^{*}=13 L^{*} \cdot\left(u^{\prime}-u_{n}^{\prime}\right), \\
& v^{*}=13 L^{*} \cdot\left(v^{\prime}-v_{n}^{\prime}\right) .
\end{aligned}
$$

4.3. CIELAB. The "CIELAB" color space (also known as "CIE

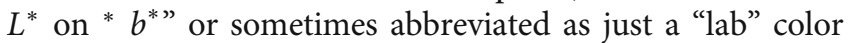
space) is a color space defined by the International Lighting Commission (CIE) in 1976. The color expresses three values: " $L$ " " for lightness from black (0) to white $(100),{ }^{*}$ from green $(-)$ to red $(+)$, and $b^{*}$ from blue (-) to yellow (+). CIELAB has been designed so that the same amount of numerical change in these values corresponds to approximately the same amount of change seen visually [7].

$$
\begin{aligned}
& L^{*}=116 f\left(\frac{Y}{Y n}\right)-16, \\
& a^{*}=500\left\{f\left(\frac{X}{X n}\right)-f\left(\frac{Y}{Y n}\right)\right\}, \\
& b^{*}=200\left\{f\left(\frac{Y}{Y n}\right)-f\left(\frac{Z}{Z n}\right)\right\} .
\end{aligned}
$$

4.4. CIESAM02. Color space CIELAB (also defined as the "CIE $L^{*} a^{*} b^{* \text { " }}$ or abbreviated in some cases as the color space "Lab") is the color space specified by the International Committee of the Lighting (CIE) in 1976. It reflects the three elements of the values that specifies the hue: $L^{*}$ for lighting from black (zero) to white (100), ${ }^{*}$ from green (-) to red (+), and $b^{*}$ from blue (-) to yellow (+). It is designed to be compatible with "CIELAB" the same amount of change in these numerical values with almost the same amount of change seen visually [8]. The general mathematical equations that determine the relationship between the elements of this system are as follows:

$$
\begin{aligned}
& \begin{array}{cc}
L & X \\
M=M_{\text {CAT02 }} & Y, \\
S & Z
\end{array} \\
& \begin{array}{lll}
0.7328 & 0.4296 & -0.1624
\end{array} \\
& \mathrm{M}_{\text {CAT02 }}=0.7036 \quad 1.6975 \quad 0.0061 \text {, } \\
& 0.0030 \quad 0.0136 \quad 0.9834 \text {, } \\
& \mathrm{Lc}=\left(\frac{Y w L w r}{Y w r L w} D+1-D\right) L^{\prime}, \\
& \mathrm{Mc}=\left(\frac{Y w M w r}{Y w r M w} D+1-D\right) M^{\prime}, \\
& \mathrm{Sc}=\left(\frac{Y w S w r}{Y w r S w} D+1-D\right) S^{\prime} .
\end{aligned}
$$

$Y w / Y w r$ represent the two bright elements that have the same color but are different from whites. Common values indicate the response of the cone to white below $(w)$ and reference illumination $(w r)$. The adjustment degree $(D)$ can be set to zero for nonadjustment (self-lighting incentive) and the unit for full adjustment (color fastness). In practice, in values ranging from 0.65 to 1.0 , the values listed can be calculated by the equation below [8]:

$$
D=F\left(1-\frac{1}{3.6} e^{-\left(L_{A}+42\right) / 92}\right)
$$

where surround " $F$ " is as defined above and " $L_{A}$ " is the adapting field luminance in $\mathrm{cd} / \mathrm{m}^{2}$.

\section{RGB Color Model}

The main colors, red, blue, and green, are originally heads Maxwell's triangle; this kind of representation of the values of the colors is essential in the visual devices because of the possibility of electronic devices from conducting mixing processes for the production of subcolors in the color images close to the natural state to ensure the image compatibility displayed on the screen.

There are other spaces derived from the RGB color space, such as "sRGB," "Adobe RGB," "ProPhoto RGB," "scRGB," and CIE RGB. 


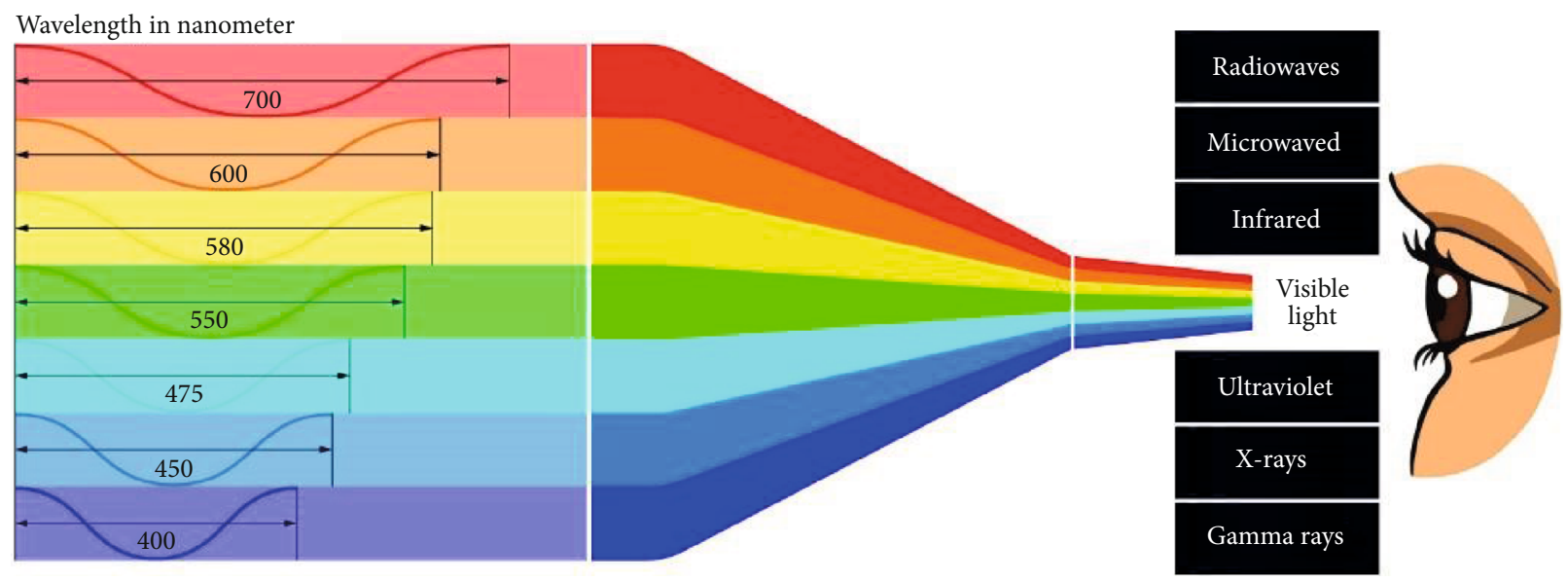

FIGURE 2: The main colors (RBG) for human vision [9].

The ideal mathematical model for describing color mixing operations with numerical values arranged by arrays of numbers, usually three or four values or color components, there is a simple linear relationship between the RGB and XYZ spaces:

$$
\begin{array}{lrrr}
\mathrm{R} & 3.240454 X & -1.537138 Y & -0.498531 Z, \\
\mathrm{G}=-0.96926 X & 1.876010 Y & 0.04155 Z, \\
\mathrm{~B} & 0.055643 X & 0.204025 Y & 1.057225 Z .
\end{array}
$$

The main colors (RBG) are suitable for human vision field (400-700 nm) as shown in Figure 2 [9]. Therefore, they are used in all kinds of visual systems such as TV broadcasting, image processing, and printing [10].

\section{The Use of Color Spaces in Television Broadcasting Systems}

There are several color television broadcasting systems, including "PAL," "SECAM," and "NTSC," which are distributed around the world. Of these systems, there are secondary systems used in digital communication and digital signal processing.

Y'IQ was previously used in television broadcasting "NTSC" (America, Japan, and elsewhere). In this system, the luma value is almost identical to the luminance [8]. "PAL" (Europe, except France, which uses SECAM) TV uses space colors "YUV" where Wi represents light, either Yu and in representing mixing operation main colors, striped "YDbDr" used by the TV "SECAM" $[9,10]$.

All of these systems can be extracted from the values of RGB which are originally suitable for human vision and thus enable the human to see the television broadcast conveniently.

\subsection{YIQ Was Formerly Used in NTSC.}

$$
\begin{array}{ccccc}
Y & 0.299 & 0.587 & 0.114 & R \\
I= & 0.5959 & -0.2746 & -0.3213 & * \\
Q & 0.2115 & -0.5227 & 0.3112 & B
\end{array}
$$

For all conversions from RGB to TV systems, it has used matrix $M$ as Figure 3 [11].

6.2. The "YUV" Color Model Is Used in the "PAL" Composite Color Video [13].

$$
\begin{array}{ccccc}
Y & 0.299 & 0.587 & 0.114 & R \\
U=-0.14713 & -0.28886 & 0.436 & * G \\
V & 0.615 & -0.51499 & -0.10001 & B
\end{array}
$$

6.3. The YDbDr Scheme Used by SECAM Television [14].

$$
\begin{array}{ccccc}
Y & 0.299 & 0.587 & 0.114 & R \\
D^{\prime} b= & -0.450 & -0.883 & 1.333 * & G^{\prime} . \\
D r & -1.333 & 1.116 & 0.217 & B
\end{array}
$$

\section{Color Printing}

Model color "CMYK" is the model chromatic main colors used that are different from the colors blue, green, and red, where the colors used are the color of cyan, magenta, yellow, and key color (black color) as in Figure 4, suitable for inks and so used in color printing devices [15].

$$
\begin{aligned}
& R^{\prime}=\frac{R}{225}, \\
& G^{\prime}=\frac{G}{225}, \\
& B^{\prime}=\frac{B}{225} .
\end{aligned}
$$

From these values, the value of colors can be calculated from the following equations: 

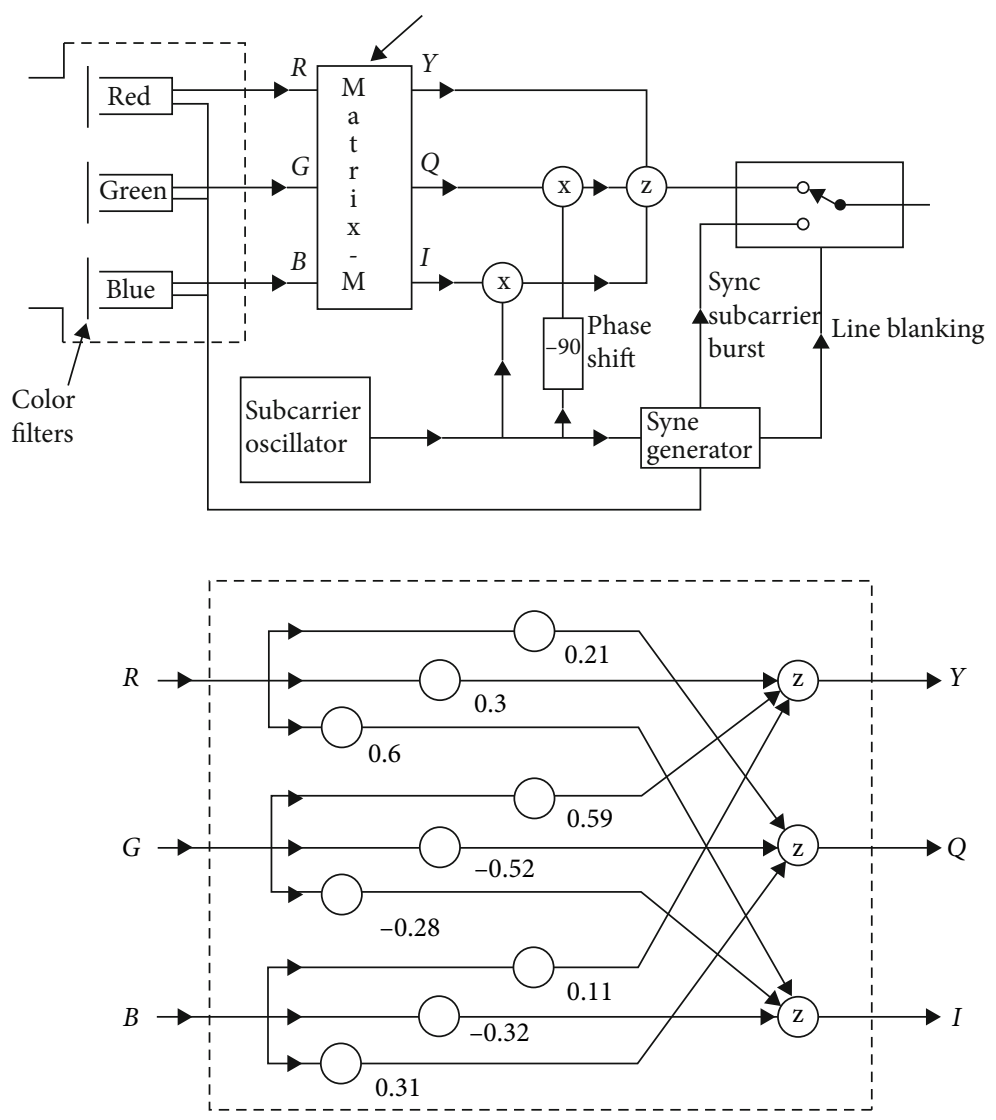

Figure 3: Conversion RGB to NTSC using matrix $M$ [12].

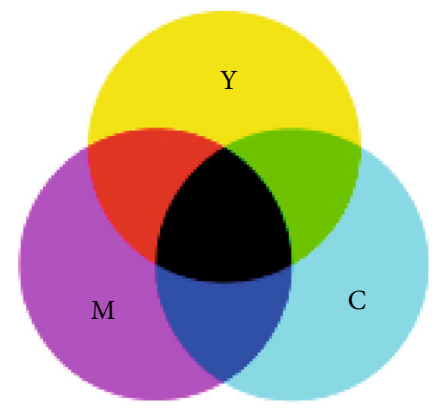

FIgURE 4: The CMYK color model [16].

$$
\begin{gathered}
K=1-\max \left(R^{\prime}, G^{\prime}, B^{\prime}\right), \\
C=\left(1-R^{\prime}-K\right)(1-K), \\
M=\left(1-G^{\prime}-K\right)(1-K), \\
Y=\left(1-B^{\prime}-K\right)(1-K),
\end{gathered}
$$

\section{The Use of Color Spaces in Digital Form and Video Compression}

YPbPr is a miniature version of "YUV." It is most commonly seen in its digital format "YCbCr," widely used in video and image compression programs such as "MPEG" and "JPEG."

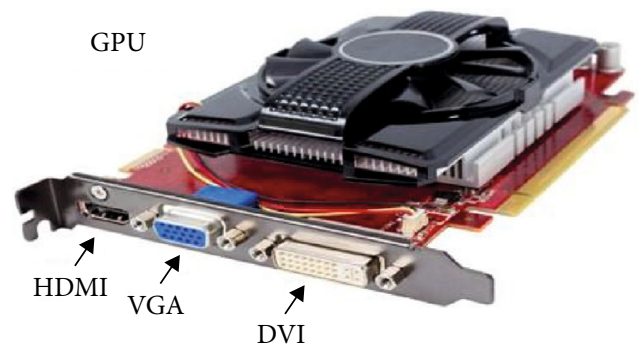

Figure 5: Graphics processing unit [19].

$\mathrm{xvYCC}$ is the digital video standard international color space adopted by the Independent Electoral Commission (IEC 61966-2-4) that it is based on the standards of "BT.709" ITU BT.601, but the series extends beyond the primary $(R / G / B)$ specified in those standards [17].

General purpose computing on graphics processing unit is the use of a graphics unit (GPU) and number processing (Figure 5), which usually handles only an account for computer images, to perform an account in processing applications traditionally by GPU CPU. Using multiple video cards in a single computer or large numbers of graphics chips, parallelizes as well as the already parallel nature of graphics processing. In addition, even a single "GPU CPU" framework provides the advantages that multiple CPUs on their own do advance due to the specialty of each chip [18]. 


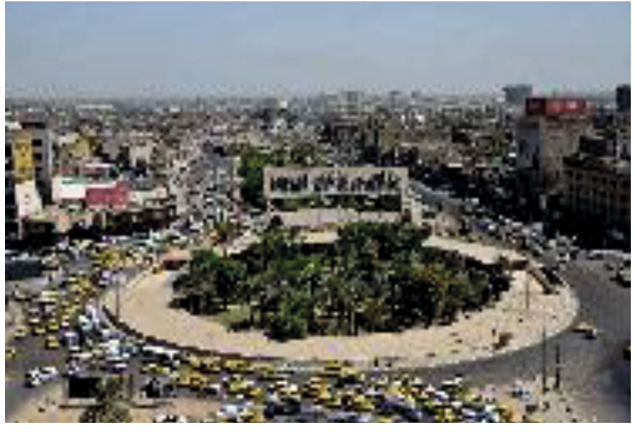

Figure 6: Full color test image.

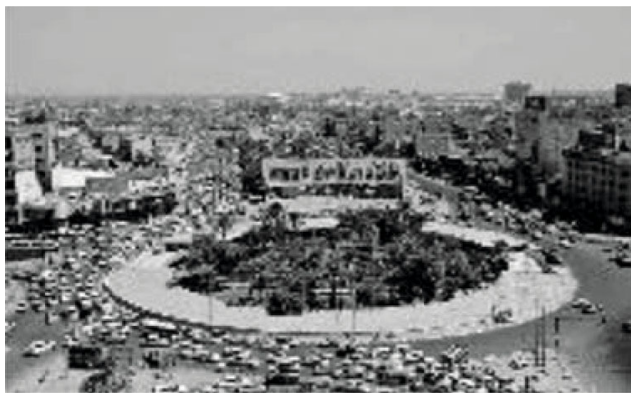

FIgURE 7: Image with coefficient $Y^{\prime}$.

\section{Matlab Simulation of Color Systems}

The simulation of this system deals with the Matlab application, which was used for color analyses that can be derived from "RGB" information provided by tools such as cameras, allowing the application to select the color image and then analyze it into space (CIE) and then to the "NTSC' system, the application outputs in different color analyses provided by a matching image test (Tahrir Square in Baghdad).

The application used is called color spaces (Figures 6-9), which is used to measure different colors of video broadcast and analyze color spaces, i.e., it has RGB representation and scale $(256 \times 256$ pixels and 24 -bit color depth); then, the conversion is displayed input to the basic spaces (RGB to Y'IQ), for all images that proved the results match the practical side with the theoretical [20].

We note from the results of the test image as in Figure 6 that the amount of the value of $Y^{\prime}$ represents the gray gradient of the image of any difference in the intensity of light between the black and white as in Figure 7, while the value of $I$ as in Figure 8 represents the chromatic part of the color mixing to complete with the value of $Q$ as in Figure 9. These mixing results are all emitted from the main space $(R, G, B)$ as in equation (9) mentioned previously, as this space is the basis of all the systems and the common factor between them [12].

\section{Results and Discussion}

The development of communication applications in the areas of image transmission, analogue video, and digital video was preceded first by an evolution in the methods of mathemati-

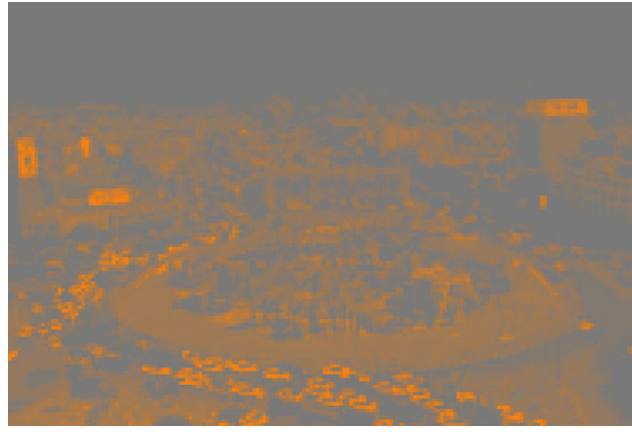

FIgURE 8: Image with coefficient $I$.

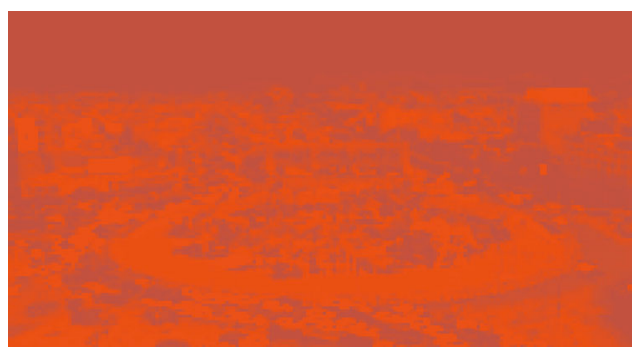

Figure 9: image with coefficient $Q$.

cal representation of color spaces so that it provides an accurate response to how to deal with the main components of the image and is the origin of the image itself, which is essentially a difference in the intensity of lighting between black and white. Gray hue and then the addition of other colors resulting from the main colors, red, green, and blue, and the rest of the colors are mixing of the main colors in numerical proportions according to the type of space representation shown previously.

In this research, the mathematical and logical analysis of many systems that use colors was done, which as previously presented can solve many problems of color compatibility between different systems, as it was found that different TV systems all meet in the space of the three main colors $(R, G, B)$. So, when converting one system to another, the space $(R, G, B)$ can be the common factor between them, so it is possible to transfer from one system to another by using that feature. In this research, it was also noticed that the printing system $(C, M, Y$, and $K$ ) suffers from inconsistencies between the color outputs in the screen during printing and the output of the image after printing, as there is a clear difference in color clarity when comparing the color image on the display in the computer and the color image on paper after it is printed on the color printer; here is the problem in how to reduce that difference and try to match the output of printing on a paper with the one on the display, for this purpose the following measures can be taken:

(1) Create electronic equipment and software to create a transition phase to reduce the problem of color contrast between the architectural design presentation and the prints 
(2) Increase the color space by creating a special (RGB) system for designers who rely on converting the image to (CMYK) when printing to reduce the effect of the difference

(3) The conversion from RGB to CMYK system will be one time because more than that will lose many original design specifications due to the loss of information in signal processing

(4) It has been observed that the use of green color frequently in many color images, especially in architectural designs, leads to a contrast in the colors between a screen and a paper, because the green area in the printing system is very weak, so it is better to reduce that color in the basic design of the image [15].

This research was carried out with the efforts of researchers with the support of the colleges to which they belong, as a part of the requirements for academic work requirements that encourage the development the field of research in line with the duties of academic teaching.

\section{Conclusions}

The technical development in color systems has led to a great diversity in the field of video communications and image processing in television broadcasting and transmission over the Internet as well as high-definition printer. Color spaces are constantly evolving due to the emergence of an intelligent generation of electronic chips that allows the production of more image processors with a wide range of color levels according to multiple mixing grades, resulting in very accurate gradations of the exact wavelengths of the color wheel, which led to a significant increase in the number of visible colors.

\section{Data Availability}

Access to the data was through the conclusions and observations taken from the sources referred to in the original research.

\section{Conflicts of Interest}

The authors declare that they have no conflicts of interest.

\section{Acknowledgments}

This research was done with the efforts of researchers, with the support of their colleges to which they belong, so the researchers thank the scientific and moral support from their colleges. All the information that the researchers obtained was through their own. The researchers belong to different colleges from different universities, all of which are Iraqi Universities (Samarra \& Mustansiriyah) with a high scientific standing and a good reputation in the scientific and academic field efforts.

\section{References}

[1] P. M. Harman, The scientific letters and papers of James Clerk Maxwell, Cambridge University, Cambridge, UK, 2002.

[2] H. S. Fairman, M. H. Brill, and H. Hemmendinger, "How the CIE 1931 color-matching functions were derived from Wright-Guild data," Color Research and Application, vol. 22, no. 1, pp. 11-23, 1997.

[3] Danny, "A review of RGB color spaces ... From Xin to R'G'B'," 2010.

[4] D. Margolis, The Canyon Conundrum and Other Adventures in the Most Powerful Color Space, Berkeley, Calif, London, 2006.

[5] J. Schanda, Colorimetry: Understanding the CIE System, Wiley Interscience, 2007.

[6] R. W. Hunt, Measuring Colour, Fountain Press, England, 3rd edition, 1998.

[7] International Color Consortium, Specification ICC.1: 2004-10 (Profile version 4.2.0.0) Image Technology Colour Management - Architecture, Profile Format, and Data Structure, International Color Consortium, 2006.

[8] A. Thwaites, C. Wingfield, E. Wieser, A. Soltan, W. D. Marslen-Wilson, and I. Nimmo-Smith, "Entrainment to the CIECAM02 and CIELAB colour appearance models in the human cortex," Vision Research, vol. 145, pp. 1-10, 2018.

[9] H. Adam, "All about RGB technology," August 2018, https:// techlarva.com/about-rgb-technology.

[10] R. W. G. Hunt, The Reproduction of Colour, Wiley-IS\&T Series in Imaging Science and Technology, Chichester UK, 6th edition, 2004.

[11] M. Sinecen, Digital Image Processing with MATLAB, Applications from Engineering with MATLAB Concepts, J. Valdman, Ed., IntechOpen, 2016.

[12] R. M. Mahmood, "Color spaces analysis for luminance \& chrominance signals AS NTSC-TV system," Journal of Engineering and Development, vol. 15, no. 4, 2011.

[13] D. L. Macadam, "Projective transformations of I. C. I. color specifications," Journal of the Optical Society of America, 1937.

[14] R. J. Crane, The Politics of International Standards: France and the Color TV War, Ablex Publishing Corporation, 1979.

[15] R. H. Yaseen, R. Mahmood, and M. Darweesh, "Color spaces representation and its role in the architectural design," International Review of Civil Engineering, vol. 9, no. 5, p. 202, 2018.

[16] D. Van Holtan, "RGB Vs CMYK," 2016, https://www .printernational.org/rgb-versus-cmyk.php.

[17] J. Hudson, 3Gb/s SDI for Transport of 1080, 3D, UHDTV1 / $4 k$ and Beyond, Professional Development SMPTE, 2013.

[18] N. Katoh, "New" extended-gamut color space for video applications; xvYCC (IEC61966-2-4), Hollywood Post Alliance, 2007.

[19] GPU History, Hitachi ARTC HD63484. The second graphics processorIEEE Computer Society.

[20] D. Silage, Digital Communication Systems Using MATLAB and Simulink, Temple University, Bookstand Publishing, 2016. 